\title{
Lettre à Ignazio Cassis
}

\section{Jean Martin}

Ancien médecin cantonal vaudois, membre de la rédaction

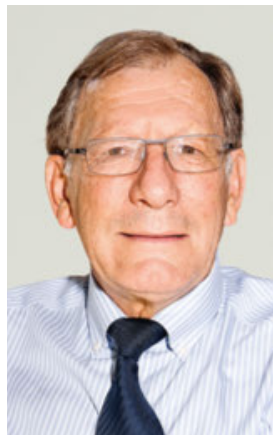

Cher confrère,

Nous nous connaissons de très longue date, nos liens d'estime et d'amitié sont forts. Tu es spécialiste FMH en médecine interne et aussi en prévention et santé publique. Tu as efficacement présidé pendant huit ans Santé Publique Suisse, notre société; tu as enseigné notre discipline. Tu présides Radix, centre national pour le développement de mesures de santé publique, et tu soutiens Fairmed, montrant ton intérêt pour les régions défavorisées. Nous œuvrons ensemble depuis cinq ans au comité de Curaviva. De plus, bien sûr, de 1996 à 2008, tu as été un excellent médecin cantonal du Tessin.

Tu as derrière toi dix ans au Conseil national et viens d'être brillamment élu à notre Gouvernement. Bravo! Le présent message aimerait, en toute modestie, te faire part de souhaits de ton vieux confrère, PLR aussi (je suis un radical historique - d'extrême centre - du canton de Vaud). Après toutes les choses attendues, «convenables» que tu as dites et sur lesquelles il faut insister pour être élu, mon espoir est que tu assumes une position d'homme d'Etat, au service de la nation; que tu agisses fortement, y compris si cela doit t'attirer des critiques, pour le mieux-être de tou(te)s et de chacun.

Les médias ont parlé de ta "glissade à droite» - et je suis d'accord avec eux, tu as pris ces dernières années des positions trop néo-libérales à mon sens. L'espace ne permet pas de s'étendre sur tes liens avec de grands assureurs au sein de Curafutura. Il y a quatre ans, tu as dû t'en expliquer devant les délégués de Curaviva, qui craignaient trop de conflits d'intérêts - crainte qui est vivement ressortie il y a peu. Je sais que tu souhaites une politique de santé rationnelle, réfléchie, qui équilibre adéquatement les "vested interests» divers. Les assureurs ne doivent pas se comporter comme des capitalistes âpres au gain, mais les médecins et d'autres partenaires doivent aussi faire preuve de bon sens et garder à l'esprit l'intérêt général - en 2012, ton retrait du comité de la FMH, suite à la position indûment corporatiste de la majorité du corps médical sur les soins intégrés, a été un acte courageux.

Tu es courageux aussi, même si cette position est à vrai dire la seule raisonnable, en t'affirmant pour une politique moderne en matière de drogue, qui va vers la régulation des produits actuellement diabolisés d'une manière qui ne tient pas compte de la réalité. Pas facile dans ce pays, mais merci d'œuvrer à faire comprendre que tous les produits psychotropes qui peuvent mener à la dépendance devraient être traités de manière semblable - que l'alcool et le tabac doivent aussi être adéquatement régulés. A propos de tabac, ceux qui se préoccupent de santé publique trouvent inacceptable, honteuse, la manière dont le groupe PLR s'est opposé au projet de loi sur les produits du tabac. Vous vous êtes montrés les serviteurs des lobbyistes qui refusent dogmatiquement de limiter l'accès à des produits qui tuent dix mille Suisses chaque année. Permets-moi ici de démonter l'argumentation de ces lobbys: ce ne sont pas les avocats de la santé qui limitent la liberté de choix des gens, ce sont les firmes du tabac, en consacrant de

Mon espoir est que tu agisses fortement, même si cela t'attire des critiques, pour le mieux-être de tou(te)s et de chacun.

grands moyens à discréditer des données scientifiques indiscutables, avec le matraquage publicitaire que nous savons. Ce sont eux les ayatollahs dangereux. Nous montreras-tu dans les années qui viennent que tu n'es pas/plus leur ami?

Un point encore, majeur: les enjeux formidables liés aux atteintes à l'environnement et au dérèglement climatique. C'est une urgence planétaire, il y va d'une vie de qualité acceptable pour les générations qui nous suivent. Merci d'œuvrer pour que la Suisse soit exemplaire à cet égard, à l'interne et au plan international.

Et je finis par l'Europe. Je reste fermement de ceux qui sont convaincus qu'une collaboration étroite avec l'Union (y compris par quelque chose comme un accord-cadre) est la seule voie possible et digne pour notre pays. Et il faut dire que, malgré les déclarations opportunistes des candidats au Conseil fédéral, le procès fait aux «juges étrangers» par les nationalistes est un mauvais procès, une manœuvre de tactique politique de plus. ${ }^{1}$

Tous mes vœux t'accompagnent, cher Ignazio. On ne gouverne qu'au centre, merci de t'en souvenir. Et merci de ne pas oublier ta maison professionnelle, la santé, la médecine et les soins. 